\title{
Effect of Sensitivity Improvement of Visible to NIR Digital Cameras on NDVI Measurements in Particular for Agricultural Field Monitoring
}

\author{
Kohei Arai ${ }^{1}$ \\ 1Graduate School of Science and Engineering \\ Saga University \\ Saga City, Japan
}

\author{
Hiroshi Sekiguchi $^{2}$ \\ 2 LSI Production Headquarters \\ Rohm Co., Ltd. \\ Kyoto City, Japan
}

\author{
Takuji Maekawa $^{2}$ \\ 2 LSI Production Headquarters \\ Rohm Co., Ltd. \\ Kyoto City, Japan
}

\author{
Toshihisa Maeda ${ }^{2}$ \\ Rohm Co., Ltd. \\ Kyoto City, Japan
}

\begin{abstract}
Effect of sensitivity improvement of Near Infrared: NIR digital cameras on Normalized Difference Vegetation Index: NDVI measurements in particular for agricultural field monitoring is clarified. Comparative study is conducted between sensitivity improved visible to near infrared camera of CuInGaSe: CIGS and the conventional camera. Signal to Noise: $\mathrm{S} / \mathrm{N}$ ratio and sensitivity are evaluated with NIR camera data which are acquired in tea farm areas and rice paddy fields. From the experimental results, it is found that $S / N$ ratio of the conventional digital camera with NIR wavelength coverage is better than CIGS utilized image sensor while the sensitivity of the CIGS image sensor is much superior to that of the conventional camera. Also, it is found that NDVI derived from the CIGS image sensor is much better than that from the conventional camera due to the fact that the sensitivity of the CIGS image sensor in red color wavelength region is much better than that of the conventional camera.
\end{abstract}

Keywords-CuInGaSe; SiCMOS; NDVI; Rice crop; Tealeaves; S/N ratio; Sensivity

\section{INTRODUCTION}

Most of the commercially available digital cameras use Silicon utilized SiCMOS or CCD for the detector. The material of these detectors is silicon. Therefore, wavelength regions are limited up to around $900 \mathrm{~nm}$ due to restriction of silicon image sensor of responsibility. Although these visible to $900 \mathrm{~nm}$ wavelength region of digital cameras are acceptable for the general purposes, there are strong demands for acquisition of camera images with visible to near infrared region in particular for biometric security system, medical check system, agricultural and forestry application fields. In order to improve the sensitivity of the detector, CuInGaSe: CIGS image sensor is developed by Rohm Co., Ltd. in Japan [1]-[7]. The sensitivity of the image sensor covers from visible to $1200 \mathrm{~nm}$ with the acceptable quantum efficiency.

One of application fields of the CIGS image sensor is vegetation monitoring. Importantly, vegetation monitoring needs wide wavelength coverage with an acceptable sensitivity. There are so many types of commercially available digital cameras. These, however, are not enough for monitoring of vegetation index which needs an acceptable sensitivity at red color wavelength and NIR wavelength regions. Usually, spectral reflectance of vegetation shows very low at the red color wavelength and quite high in the NIR region. Due to the fact that sensitivity of the commercially available digital cameras is not enough in NIR region while the reflectance of vegetations in red color wavelength is low, it would be better to improve the sensitivity of the digital camera in NIR wavelength region together with red color region. The CIGS image sensor gives one of solutions for solving the above mentioned problem.

Tea farm areas and rice paddy fields are selected for showing an effectiveness of the CIGS image sensor in particular for improving sensitivity in red to near infrared regions. The most important thing for tealeaves monitoring is NDVI estimation [8]-[20]. Amino acid contents containing in tealeaves depends on NDVI. Amino acid rich tealeaves taste good. Also, tealeaf growing stage monitoring needs fiber content estimation. Depending on growing stage, fiber content is getting large. Therefore, it is possible to estimate fiber content in tealeaves.

Meanwhile, protein content in rice crops is highly correlated with nitrogen content in rice leaves [21]-[26]. Protein content rich rice crops taste bad. Therefore, it is possible to estimated rice crop quality once protein content in rice crops is estimated. Nitrogen content in rice leaves depends on rice leaf reflectance. Therefore, it is capable to estimate protein content in rice crops through estimation of nitrogen content in rice leaves which is done with visible to near infrared camera data.

In order to estimate NDVI, leaf reflectance has to be measured in red and near infrared wavelength regions. The sensitivity of the CIGS image sensor in these wavelength regions is much better than those of the conventional digital cameras which utilized SiCMOS or CCD with silicon 
materials. Therefore, it is expected that NDVI estimation accuracy is improved. More than that, the sensitivity of the CIGS image sensor is better than that of the conventional digital cameras. This paper clarified these improvements quantitatively.

The next section describes the specification of the CIGS image sensor followed by the method and procedure of the experiments Then experimental results are described followed by conclusions with some discussions.

\section{Cigs IMAge Sensor}

\section{A. Specific Features of the CIGS Image Sensor}

One of the specific features of the CIGS image sensor is wide spectral coverage ranges from 430 to $1025 \mathrm{~nm}$ with the sensitivity level (Quantum Efficiency) of 30. The wavelength coverage of the conventional SiCMOS image sensor ranges from 400 to $750 \mathrm{~nm}$. Fig. 1 shows wavelength coverage of the CIGS image sensor and the conventional SiCMOS image sensor while Fig. 2 shows outlook of the CIGS image sensor.

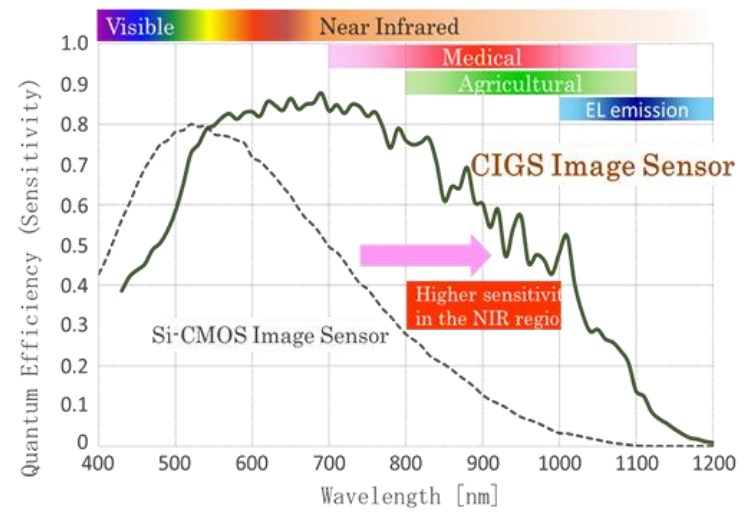

Fig. 1. Wavelength coverage of the CIGS and the conventional SICMOS image sensors

Fig.3 shows structural difference between the CIGS image sensor and the conventional SICMOS sensor. In particular, detector surface reflectance of the CIGS image sensor is much lower than that of the conventional SiCMOS image sensor. Also, aperture ratio of the CIGS image sensor is much higher than that of the conventional SiCMOS image sensor which results in high sensitivity in visible to near infrared wavelength regions.

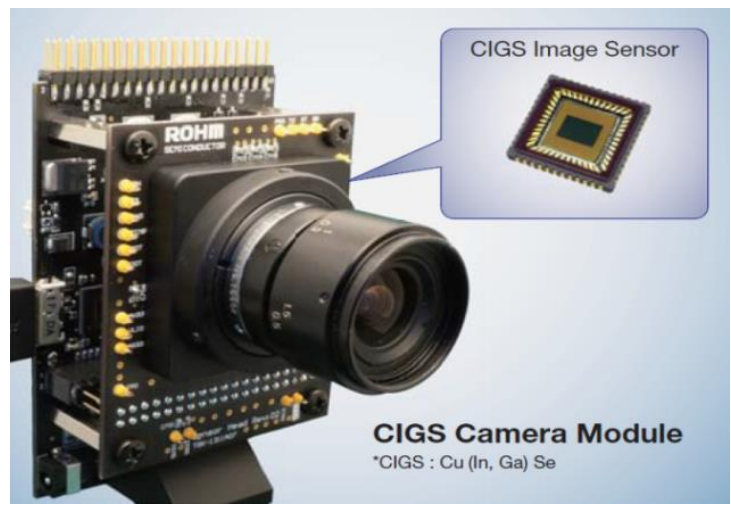

Fig. 2. Outlook of the CIGS image sensor

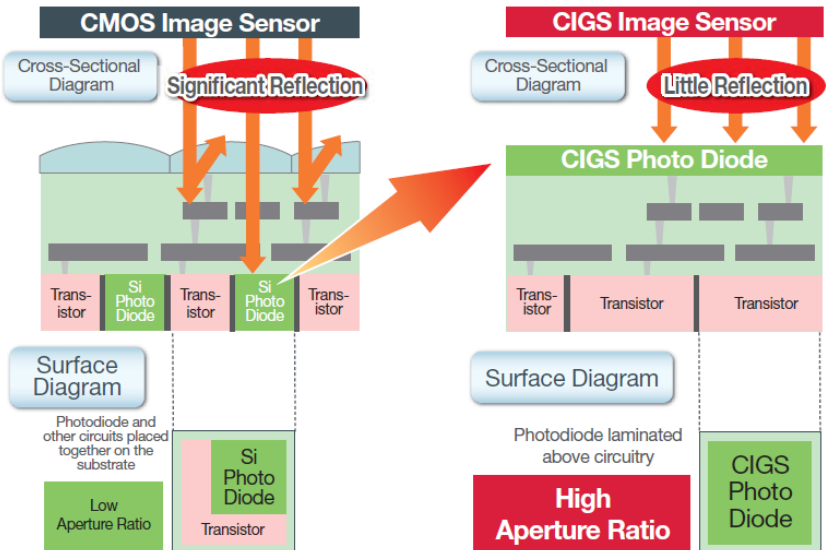

Fig. 3. Structural difference between the CIGS image sensor and the conventional SICMOS sensor

\section{B. Specific Applications of the CIGS Image Sensor}

Fig.4 (a) and (b) shows examples of applications of the CIGS image sensor for biometric sensors and for vegetation monitoring, respectively. Vein patterns of human hands can be detected by the CIGS image sensor as a biometric sensor for identification of the registered persons for security reason. On the other hand, vegetation monitoring can be done with the CIGS image sensor as shown in Fig.4 (b) because the reflectance of vegetation is very high in near infrared wavelength region.

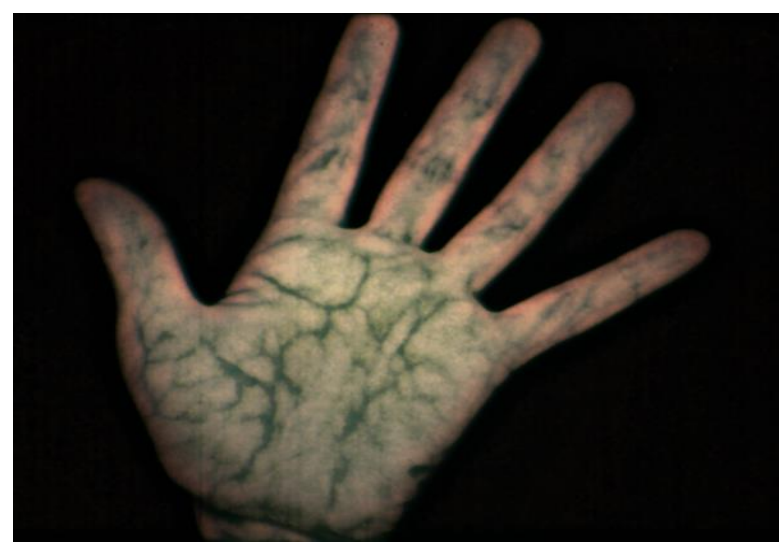

(a)Biometric sensor

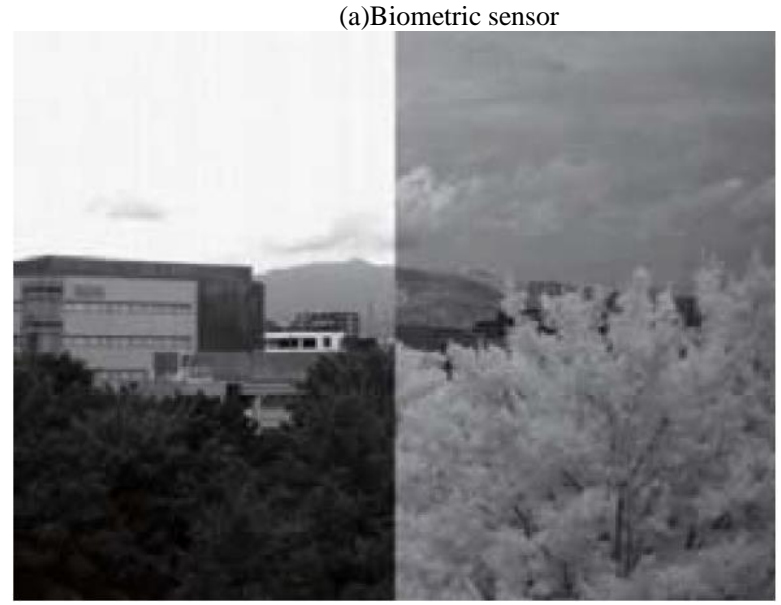

(b) Vegetation

Fig. 4. Examples of the CIGS image sensor 
Fig.4 (b) left shows the outdoor scenery of vegetation acquired by CIGS image sensor without any filter while Fig.4 (b) right shows that with visible wavelength coverage cut filter. It is quite obvious that vegetated areas are brighter for the CIGS image sensor image than those areas for the CIGS image sensor with visible wavelength coverage cut filter image (NIR image).

\section{EXPERIMENTS}

\section{A. Method and Procedure}

Signal to Noise ratio: $\mathrm{S} / \mathrm{N}$ ratio is evaluated by assuming the mean of the small portion of the acquired image where is seemed to be homogeneous pixel values must be the signal and by assuming the standard deviation of the same area of image is the noise. On the other hand, sensitivity can be evaluated by taking ratio between mean values of the different homogeneous portions of images.

Comparisons of $\mathrm{S} / \mathrm{N}$ ratio and sensitivity are carried out between the CIGS image sensor and the conventional SiCMOS image sensor of Canon S100 with the replaced NIR filter to the originally blue filter. Spectral response of the S100 camera used is shown in Fig.5.

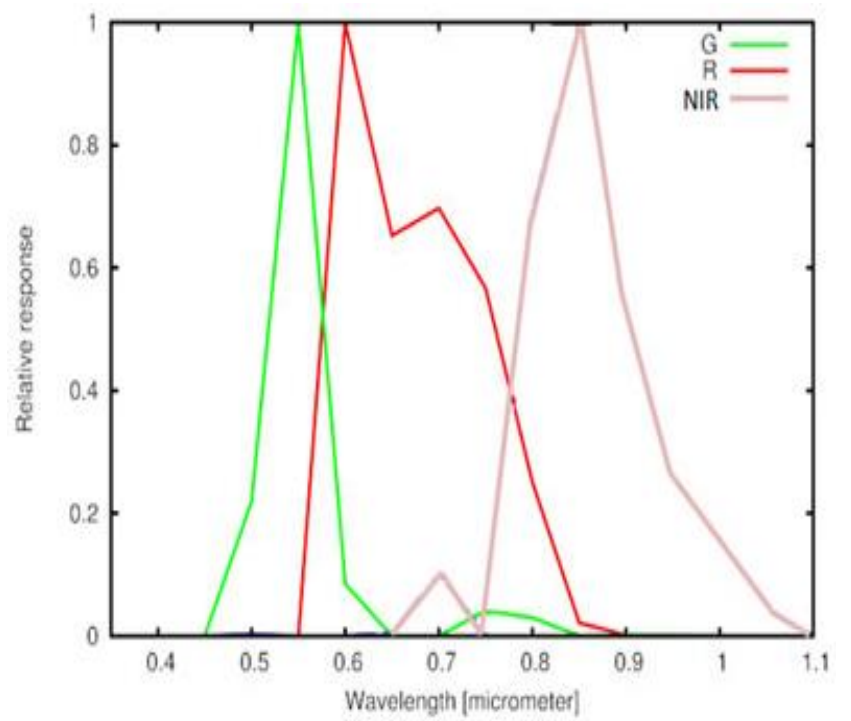

Fig. 5. Spectral Response of S100 Camera of which Blue Filter is Changed to NIR filter

\section{B. Intensive Study Araeas}

The experiments are conducted at the tea farm areas of the Saga Prefectural Institute of Tea: SPIT which is situated in Ureshino city, Saga Japan and the Saga Prefectural Institute of Agriculture: SPIA which is situated in Saga city, Saga Japan. Outlooks of SPIT and SPIA are shown in Fig.6 (a) and (b), respectively. The third tea farm field at the North tea farm areas of Yabukita Tea at SPIT is selected as tealeaf example while Hiyokumochi of sticky rice paddy field is also chosen as rice leaves example.

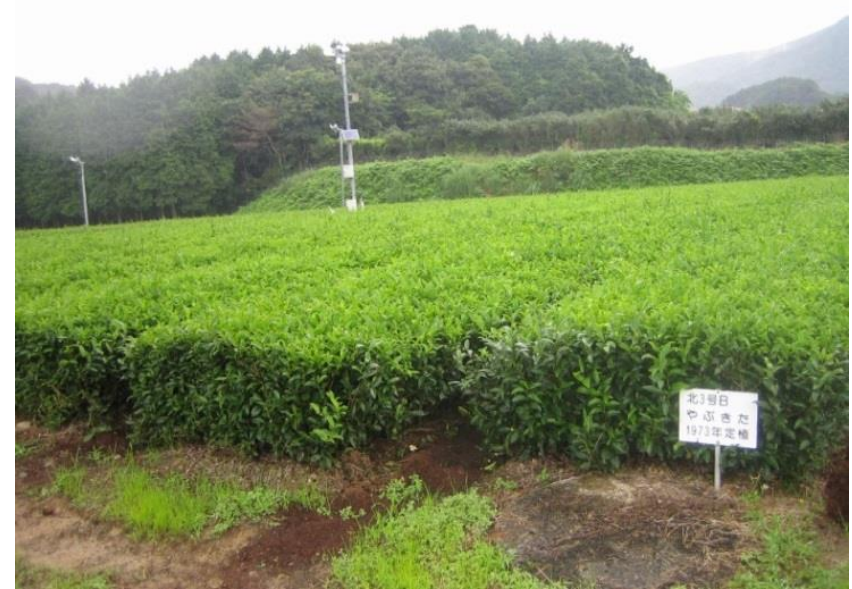

(a)SPIT

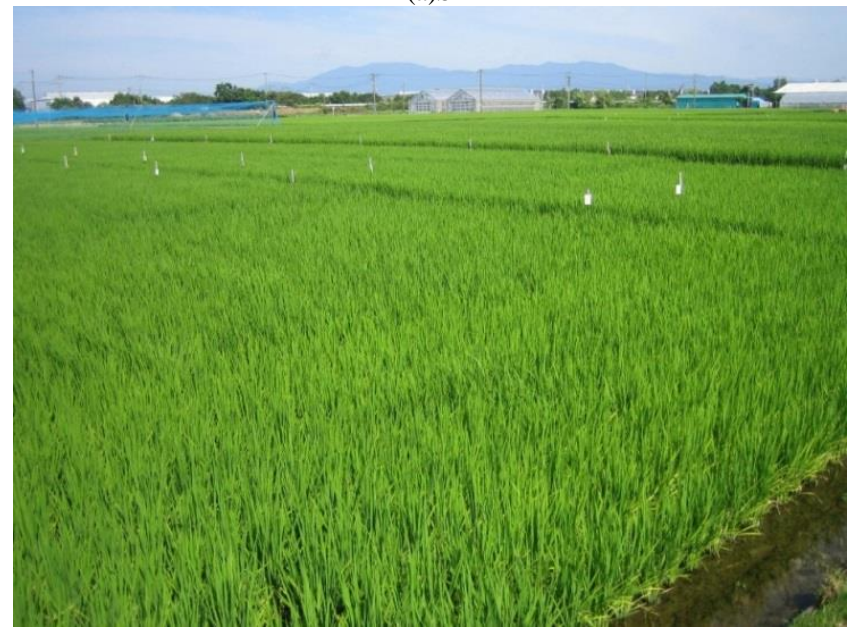

(b)SPIA

Fig. 6. Outlooks of the SPIT and SPIA

\section{S/N Ratio and Sensitivity Evaluations}

Fig.7 (a) shows top view of the acquired image of a small portion of the Yabukita Tea field on October 42015. Meanwhile, Fig.7 (b) shows the acquired image with the CIGS image sensor of the tiny portion of a piece of tealeaf together with histogram of the rectangle area of the tiny portion $(\mathrm{m} 1$ and s1 denotes the mean and the standard deviation of this portion of image, respectively) while Fig.7 (c) shows that of the different rectangle portion of the tiny portion ( $\mathrm{m} 2$ and s2 denotes the mean and the standard deviation of this portion of image, respectively). On the other hand, Fig.7 (d) shows the acquired image with the S100 camera of the almost same portion of the tealeaf ( $\mathrm{m} 3$ and $\mathrm{s} 3$ denotes the mean and the standard deviation of this portion of image, respectively) while Fig.7 (e) shows that of the different portion of the tealeaf on October 72015 (m4 and s4 denotes the mean and the standard deviation of this portion of image, respectively). All these images are acquired by the CIGS image sensor and the S100 image sensor with NIR filter. 


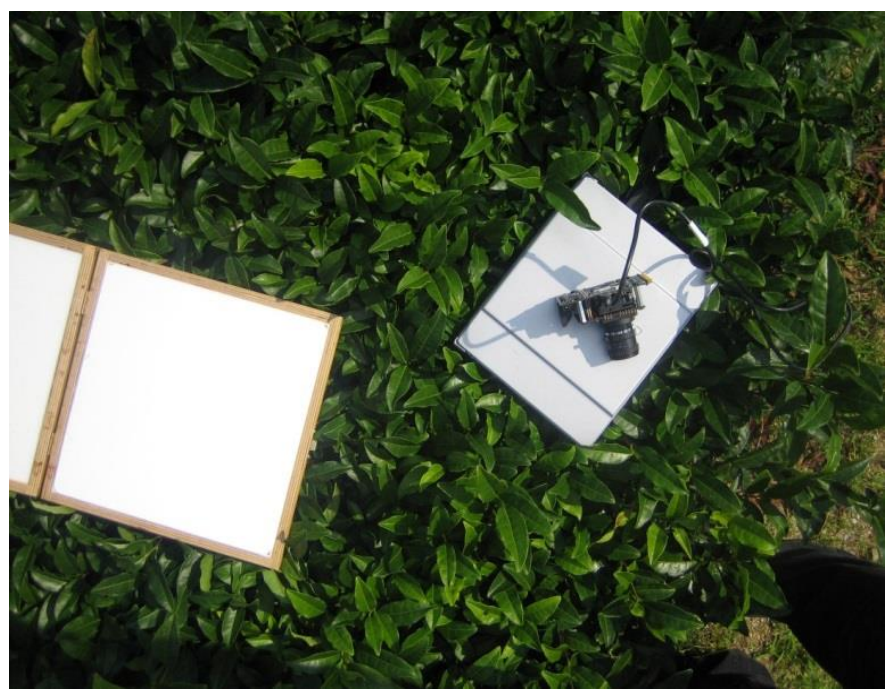

(a)Top view of Yabukita tealeaf

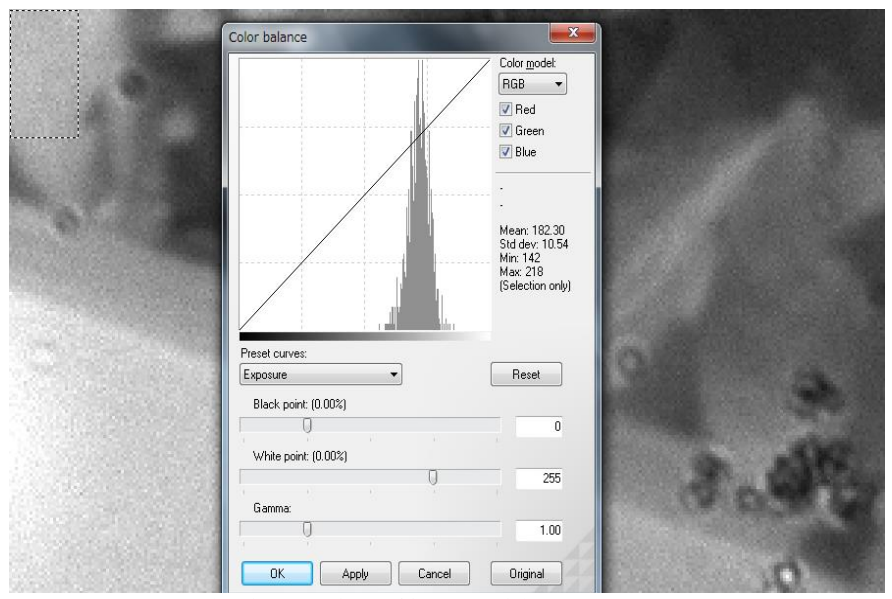

(b)Tiny portion of image with CIGS camera

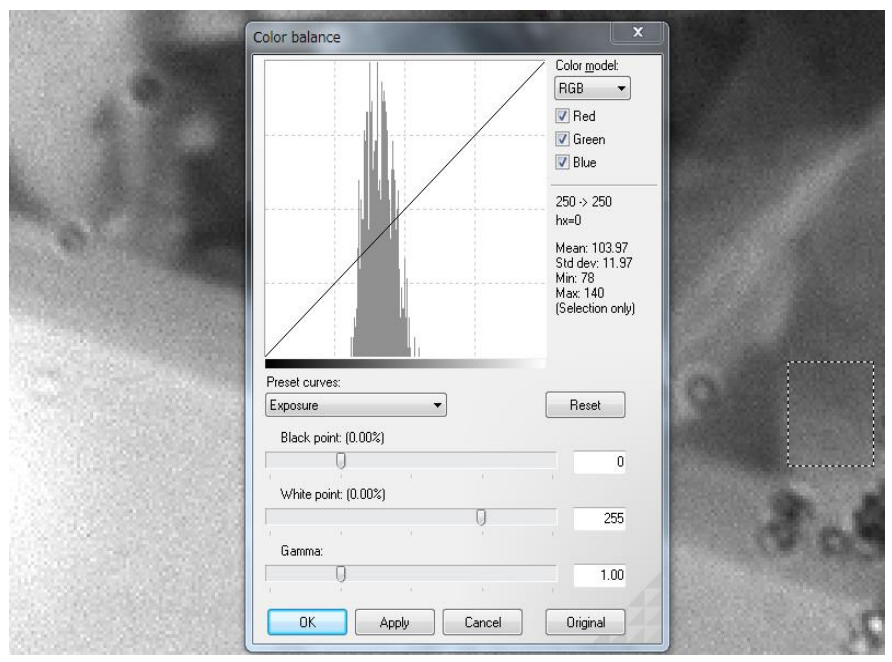

(c)Different portion of image with CIGS camera

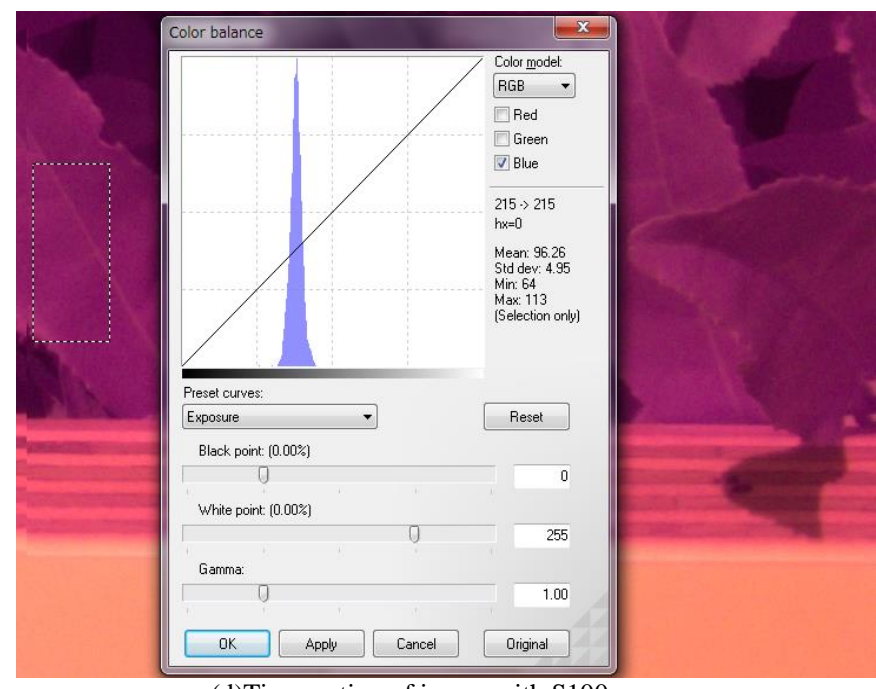

(d)Tiny portion of image with S100 camera

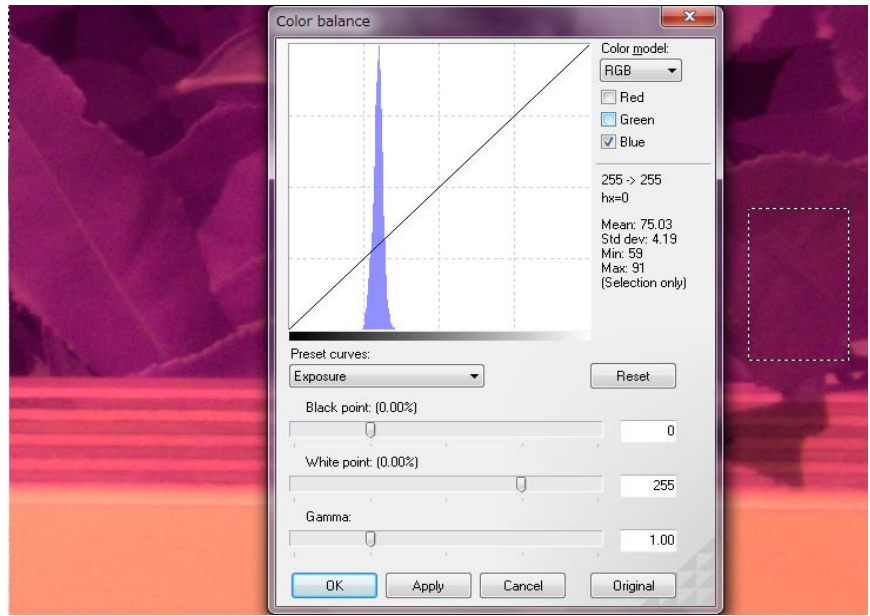

(e)Different portion of image with S100 camera

Fig. 7. Acquired images of Yabukita tealeaves with CIGS and S100 cameras

Fig.8 (a) shows slant view of the acquired image of a small portion of the Hiyokumochi rice paddy field. Meanwhile, Fig.8 (b) shows the acquired image with the CIGS image sensor of the tiny portion of a piece of rice leaf together with histogram of the rectangle area of the tiny portion (M1 and S1 denotes the mean and the standard deviation of this portion of image, respectively) while Fig.8 (c) shows that of the different rectangle portion of the tiny portion (M2 and S2 denotes the mean and the standard deviation of this portion of image, respectively). On the other hand, Fig.8 (d) shows the acquired image with the S100 camera of the almost same portion of the rice leaf (M3 and S3 denotes the mean and the standard deviation of this portion of image, respectively) while Fig.8 (e) shows that of the different portion of the rice leaf (M4 and S4 denotes the mean and the standard deviation of this portion of image, respectively). 


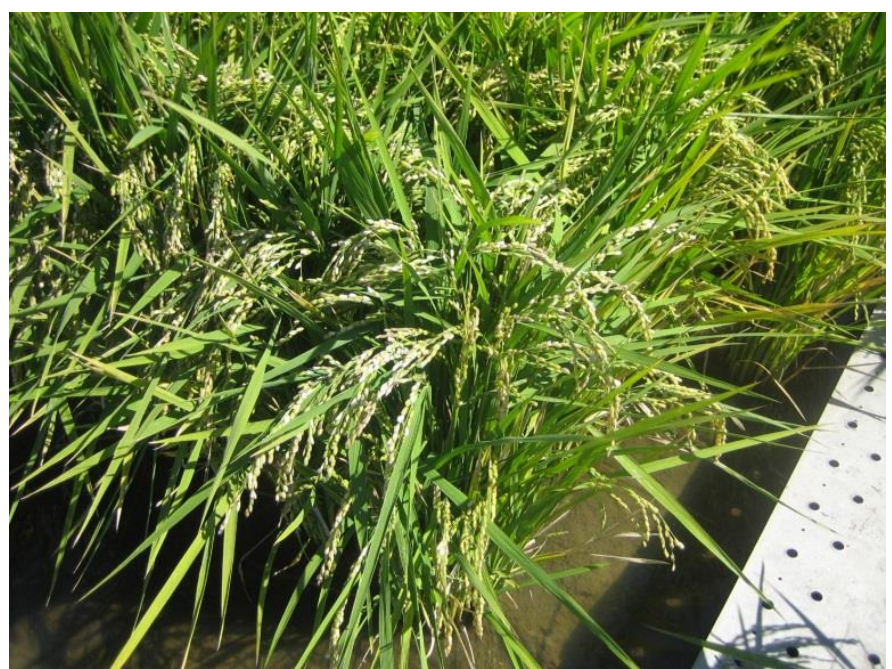

(a)Slant view

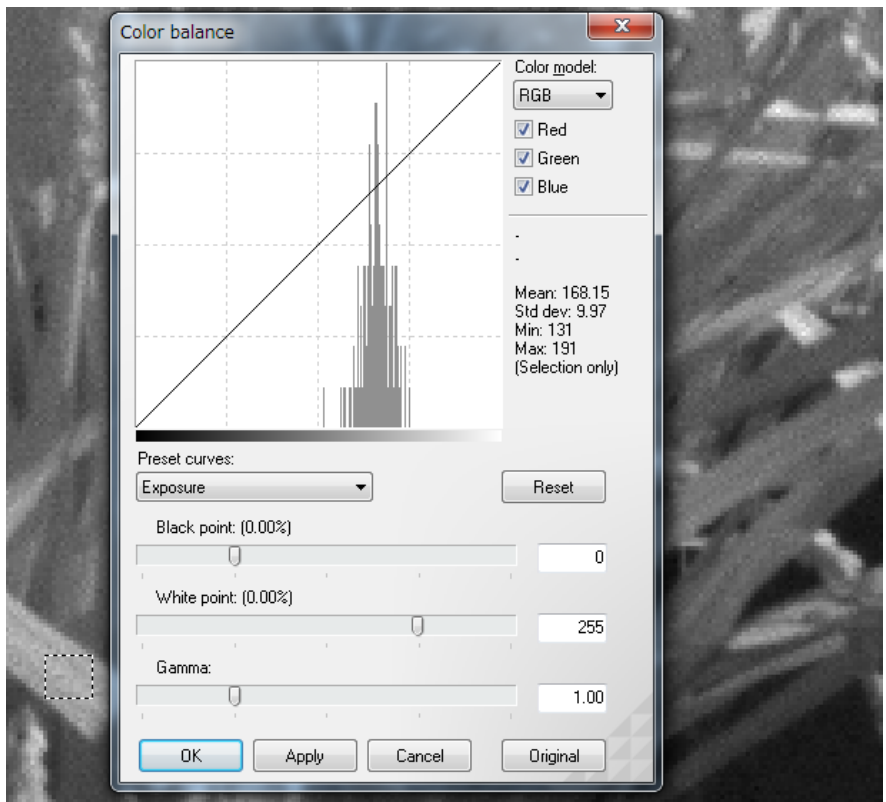

(b)Tiny portion of image with CIGS

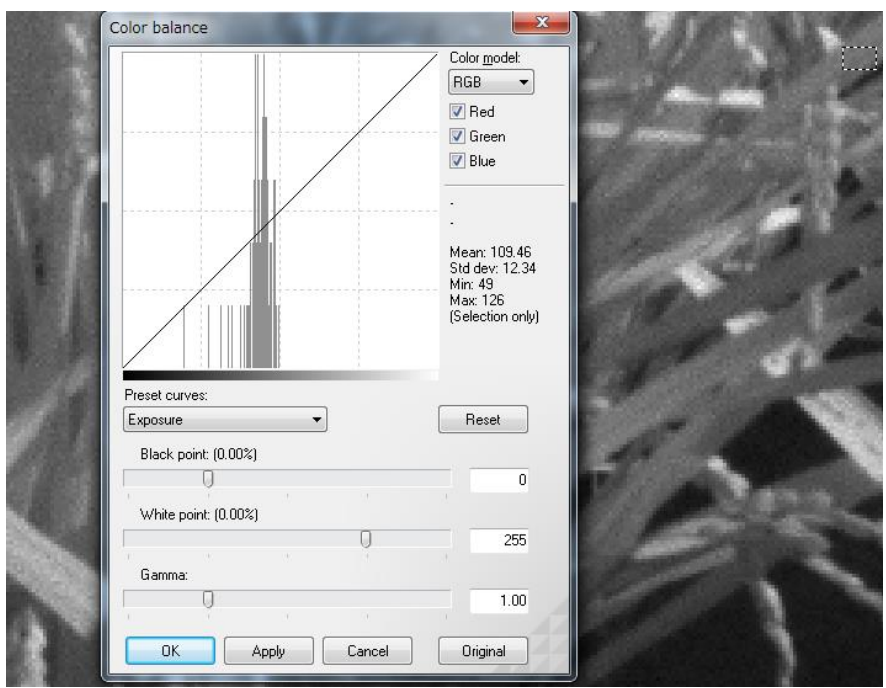

(c)Different portion of image with CIGS

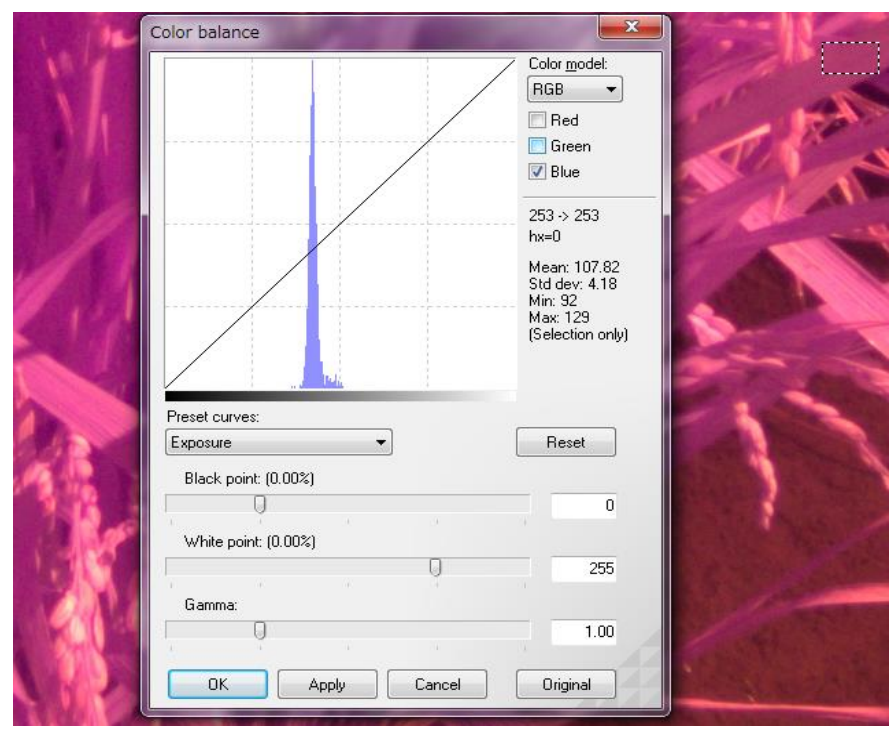

(d)Tiny portion of image with S100

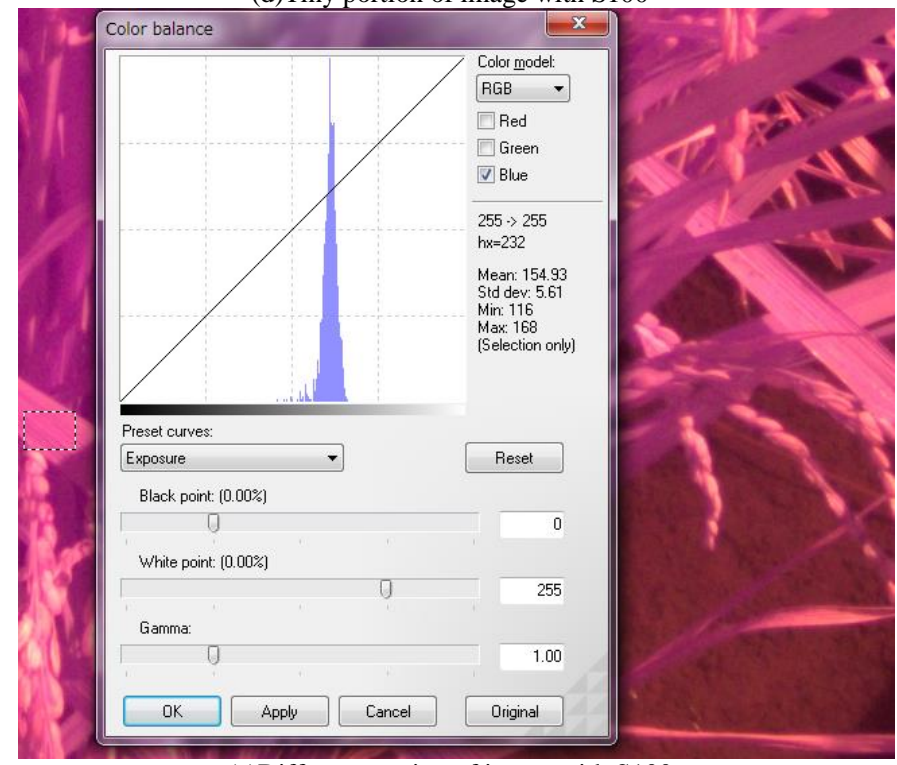

(e)Different portion of image with S100

Fig. 8. Acquired images of Hiyokumochi rice leaves with CIGS and S100 cameras

From these mean values and standard deviations, the normalized sensitivities and the normalized $\mathrm{S} / \mathrm{N}$ ratios can be calculated as follows,

\section{a) Yabukita tealeaf}

CIGS: Normalized Sensitivity:m1/m2=182.3/103.97=1.753 Normalized $\quad \mathrm{S} / \mathrm{N}=\mathrm{m} 1 / \mathrm{s} 1=182.3 / 10.54=17.296(24.76 \mathrm{~dB})$, $\mathrm{m} 2 / \mathrm{s} 2=103.97 / 11.97=8.686(18.78 \mathrm{~dB})$

S100: Normalized Sensitivity: $\mathrm{m} 3 / \mathrm{m} 4=96.26 / 75.03=1.283$ Normalized $\quad \mathrm{S} / \mathrm{N}=\mathrm{m} 3 / \mathrm{s} 3=96.26 / 4.55=20.277(26.14 \mathrm{~dB})$, $\mathrm{m} 4 / \mathrm{s} 4=75.03 / 4.19=17.907(25.06 \mathrm{~dB})$

b) Hiyokumochi rice leaf

CIGS: Normalized Sensitivity:M1/M2 $=168.15 / 109.46=1.536$ Normalized $\quad$ S/N:M1/S1=168.15/9.97=16.866(24.54dB), $\mathrm{M} 2 / \mathrm{S} 2=109.46 / 12.34=8.87(18.96 \mathrm{~dB})$ 
S100: Normalized Sensitivity:M3/M4=154.93/107.82=1.437 Normalized $\quad \mathrm{S} / \mathrm{N}: \mathrm{M} 3 / \mathrm{S} 3=154.93 / 5.61=27.617(28.82 \mathrm{~dB})$, $\mathrm{M} 4 / \mathrm{S} 4=107.82 / 4.18=25.794(28.23 \mathrm{~dB})$

As the results, it may say that sensitivity of the CIGS image sensor is 10 to $50 \%$ better than that of the S100 image sensor while $\mathrm{S} / \mathrm{N}$ ratio of the $\mathrm{S} 100$ image sensor is 50 to $100 \%$ better than that of the CIGS image sensor.

\section{NDVI Estimation Accuracy Evaluations}

Measured spectral reflectance of Yabukita tealeaves and Hiyokumochi rice leaves on October 4 and 7 are shown in Fig.9 (a) and (b), respectively.

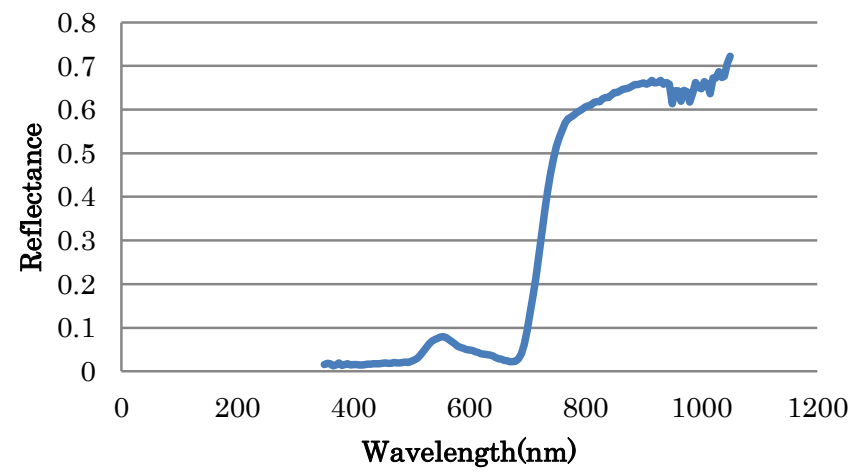

(a)Yabukita Tealeaves

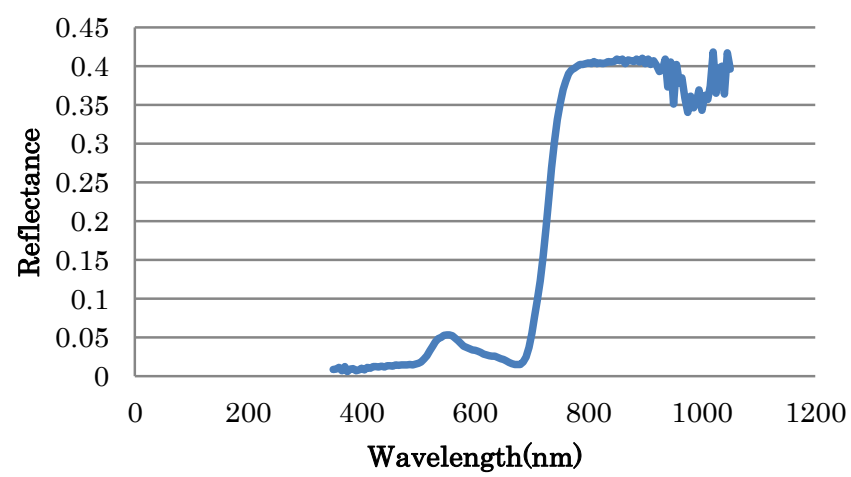

(b)Hiyokumochi Rice Leaves

Fig. 9. Spectral reflectance of the Yabukita tealeaves and Hiyokumochi rice leaves

From the spectral reflectance and the measured $\mathrm{S} / \mathrm{N}$ ratios, it is capable to predict NDVI estimation accuracy improvement by the improvement of $\mathrm{S} / \mathrm{N}$ ratio as shown in Table 1. From the $\mathrm{S} / \mathrm{N}$ ratio, it becomes available to calculate noise level. By considering the calculated noise, NDVI estimation accuracy can be evaluated as shown in Table 1.

In these cases, plus minus $10 \%$ of noise levels for each red and NIR wavelength regions are added in the NDVI equation. Then as shown in Table $1,-9$ to $8 \%$ of NDVI is deviated from the NDVI without $10 \%$ of noise for tealeaves while -2 to $2 \%$ of NDVI is deviated from the NDVI without $10 \%$ of noise for rice leaves (red colored values show improvement of NDVI calculations due to $10 \%$ improvement of $\mathrm{S} / \mathrm{N}$ of the CIGS image sensor in comparison to the S100 image sensor.
TABLE I. IMPROVEMENT OF NDVI ESTIMATION ACCURACY BY IMPROVEMENT OF S/N RATIO

\begin{tabular}{|l|l|l|l|l|l|}
\hline Tealeaf & Reflectance & S/N(dB) & Noise & NDVI+ & NDVI- \\
\hline $870 \mathrm{~nm}$ & 0.61 & 24.28 & 0.0373 & 0.8485 & \\
\hline $600 \mathrm{~nm}$ & 0.05 & 25.04 & 0.0028 & 0.8492 & 0.8477 \\
\hline Rice leaf & & & & 0.07988 & -0.0903 \\
\hline $870 \mathrm{~nm}$ & 0.4 & 31.95 & 0.0101 & 0.9048 & \\
\hline $600 \mathrm{~nm}$ & 0.02 & 32.58 & 0.00047 & 0.9049 & 0.90456 \\
\hline & & & & 0.01713 & -0.01802 \\
\hline
\end{tabular}

Quantum efficiency in red wavelength is 0.75 for the S100 image sensor while that for the CIGS image sensor is 0.81 . On the other hand, quantum efficiency in NIR $(870 \mathrm{~nm})$ is 0.19 for the S100 image sensor while that of the CIGS image sensor is 0.63 . Therefore, $\mathrm{S} / \mathrm{N}$ influence of quantum efficiency difference on NDVI calculation is as follows,

$$
\begin{array}{r}
\text { (CIGS): }(\mathrm{N} \pm 1 / 0.19-\mathrm{R} \pm 1 / 0.75) /(\mathrm{N} \pm 1 / 0.19+\mathrm{R} \pm 1 / 0.75) \\
\text { ( }) \\
\text { (S100): }(\mathrm{N} \pm 1 / 0.63-\mathrm{R} \pm 0.81) /(\mathrm{N} \pm 1 / 0.63+\mathrm{R} \pm 1 / 0.81)
\end{array}
$$

where $\mathrm{N}$ and $\mathrm{R}$ denotes the signal level in NIR and red wavelength regions, respectively. Fig. 10 shows the calculation results. When $\mathrm{S} / \mathrm{N}$ ratio at red wavelength region is improved by $10 \%$, NDVI is not so changed and improved.

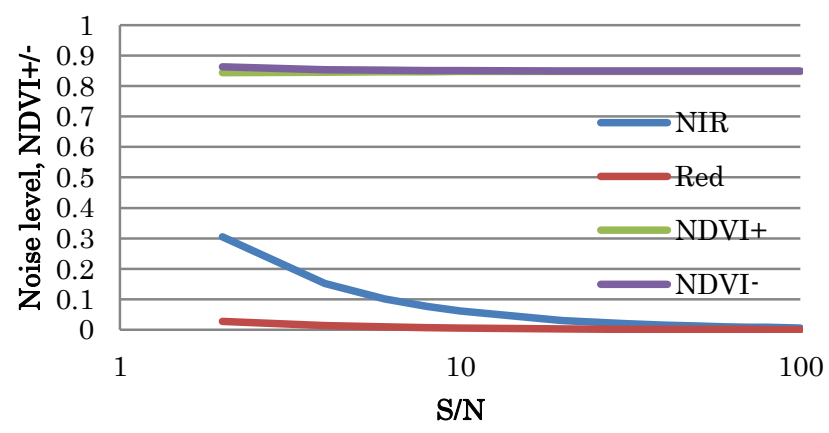

(a) $10 \%$ improvement

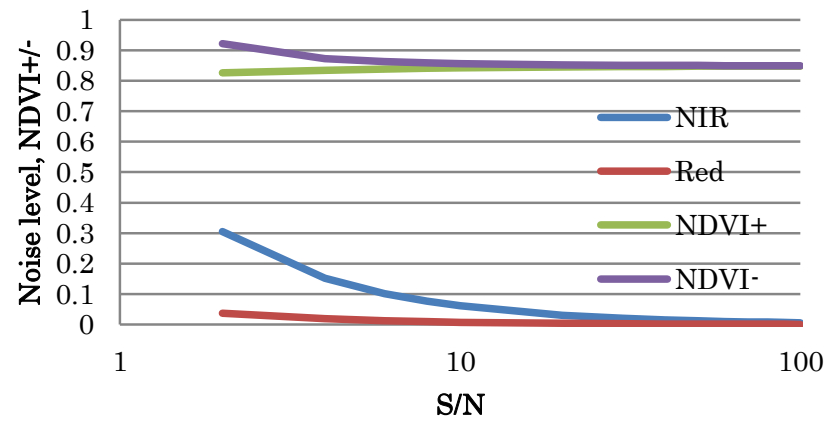

(b) $50 \%$ improvement

Fig. 10. Noise level and NDVI improvement for the cases that $\mathrm{S} / \mathrm{N}$ ratio at red wavelength region is improved by 10 and $50 \%$

Meanwhile, when $\mathrm{S} / \mathrm{N}$ ratio at red wavelength region is improved by $50 \%$, then NDVI is improved by $10 \%$. More than that, quantum efficiency of the CIGS image sensor is 7 times and twice much higher than those of S100 image sensor in NIR and red wavelength regions, respectively. Therefore, it is 
expected that estimated NDVI is 10 to $50 \%$ accurate.

\section{CONCLUSION}

Effect of sensitivity improvement of Near Infrared: NIR digital cameras on Normalized Difference Vegetation Index: NDVI measurements in particular for agricultural field monitoring is clarified. Comparative study is conducted between sensitivity improved visible to near infrared camera of CuInGaSe: CIGS and the conventional camera.

Signal to Noise: $\mathrm{S} / \mathrm{N}$ ratio and sensitivity are evaluated with NIR camera data which are acquired in tea farm areas and rice paddy fields. From the experimental results, it is found that $\mathrm{S} / \mathrm{N}$ ratio of the conventional digital camera with NIR wavelength coverage is better than CIGS utilized image sensor while the sensitivity of the CIGS image sensor is much superior to that of the conventional camera. Also, it is found that NDVI derived from the CIGS image sensor is much better than that from the conventional camera due to the fact that the sensitivity of the CIGS image sensor in red color wavelength region is much better than that of the conventional camera.

\section{ACKNOWLEDGEMENTS}

Authors would like to thank Dr. Hideo Miyazaki of Saga Prefectural Institute of Tea and Dr. Osamu Shigetomi of Saga Prefectural Institute of Agriculture and their research staff for their efforts to conduct the experiments and valuable discussions.

\section{REFERENCES}

[1] Matsushima, O., Miyazaki, K., Takaoka, M., Maekawa, T., Sekiguchi, H., Fuchikami, T., ... \& Niki, S. (2008, December). A high-sensitivity broadband image sensor using CuInGaSe 2 thin films. In Electron Image sensors Meeting, 2008. IEDM 2008. IEEE International (pp. 1-4). IEEE.

[2] K. Miyazaki, O. Matsushima, M. Moriwake, H. Takasu, S. Ishizuka, K. Sakurai, A. Yamada and S. Niki:Thin Solid Films517(2008) 7.

[3] Minoura, S., Maekawa, T., Kodera, K., Nakane, A., Niki, S., \& Fujiwara, H. (2015). Optical constants of $\mathrm{Cu}$ (In, Ga) Se2 for arbitrary $\mathrm{Cu}$ and Ga compositions. Journal of Applied Physics, 117(19), 195703.

[4] Hara, T., Maekawa, T., Minoura, S., Sago, Y., Niki, S., \& Fujiwara, H. (2014). Quantitative Assessment of Optical Gain and Loss in Submicron-Textured C u I n 1- x G a x S e 2 Solar Cells Fabricated by Three-Stage Coevaporation. Physical Review Applied, 2(3), 034012.

[5] A.M. Gabor, J. R. Tuttle, D. S. Albin, M. A. ContreasandR. Noufi:Appl. Phys. Lett.65(1944) 198-200.

[6] N. Kohara, T. Negami, M. Nishitani and T. Wada:Jpn. J. Appl. Phys. 34(1995) 1141-1144.

[7] K. Sakurai, R. Hunger, R. Scheer, C. A. Kaufmann, A. Yamada, T. Baba, Y. Kimura, K. Matsubara, P. Fons, H. NakanishiandS. Niki: Prog. Photovolt. Res. Appl.12(2004) 2.

[8] Kohei Arai, Method for estimation of grow index of tealeaves based on Bi-Directional reflectance function: BRDF measurements with ground based network cameras, International Journal of Applied Science, 2, 2, $52-62,2011$.

[9] Kohei Arai, Wireless sensor network for tea estate monitoring in complementally usage with Earth observation satellite imagery data based on Geographic Information System(GIS), International Journal of Ubiquitous Computing, 1, 2, 12-21, 2011.

[10] Kohei Arai, Method for estimation of total nitrogen and fiber contents in tealeaves with ground based network cameras, International Journal of Applied Science, 2, 2, 21-30, 2011.

[11] Kohei Arai, Monte Carlo ray tracing simulation for bi-directional reflectance distribution function and grow index of tealeaves estimation, International Journal of Research and Reviews on Computer Science, 2, 6, 1313-1318, 2011
[12] K.Arai, Fractal model based tea tree and tealeaves model for estimation of well opened tealeaf ratio which is useful to determine tealeaf harvesting timing, International Journal of Research and Review on Computer Science, 3, 3, 1628-1632, 2012.

[13] Kohei Arai, Hideo Miyazaki, Masayuki Akaishi, Tea tree vitality evaluation method and appropriate harvesting timing determination method based on visible and near infrared camera data, Journal of Japan Society of Photogrammetry and Remote Sensing, 51, 1, 38-45, 2012

[14] Kohei Arai, Method for tealeaves quality estimation through measurements of degree of polarization, leaf area index, photosynthesis available radiance and normalized difference vegetation index for characterization of tealeaves, International Journal of Advanced Research in Artificial Intelligence, 2, 11, 17-24, 2013.

[15] K.Arai, Optimum band and band combination for retrieving total nitrogen, water, and fiber in tealeaves through remote sensing based on regressive analysis, International Journal of Advanced Research in Artificial Intelligence, 3, 3, 20-24, 2014.

[16] Kohei Arai, Kyushu small satellite for remote sensing (QSAT/EOS) and value added tealeaves "Eisei-no-megumi Ureshino-cha", Journal of Society for Instrument Control Engineering of Japan, 53, 11, 988-996, 2014

[17] Kohei Arai, Yoshihiko Sasaki, Shihomi Kasuya, Hideto Matsuura, Appropriate tealeaf harvest timing determination based on NIR images of tealeaves, International Journal of Information Technology and Computer Science, 7, 7, 1-7, 2015

[18] Kohei Arai, Yoshihiko Sasaki, Shihomi Kasuya, Hideo Matsuura, Appropriate harvest timing determination referring fiber content in tealeaves derived from ground based NIR camera images, International Journal of Advanced Research on Artificial Intelligence, 4, 8, 26-33, 2015.

[19] Kohei Arai and Long Lili, BRDF model for new tealeaves on old tealeaves and new tealeaves monitoring through $\mathrm{B}$ RDF measurement with web cameras, Abstract of the 50th COSPAR(Committee on Space Research/ICSU) Congress, A3.1-0008-08 ,992, Montreal, Canada, July, 2008

[20] Kohei Arai, Estimation method for total nitrogen and fiber contents in tealeaves as well as grow index of tealeaves and tea estate monitoring with network cameras, Proceedings of the IEEE Computer Society, Information Technology in Next Generation, ITNG, 595-600, 2009

[21] Kohei Arai, Osamu Shigetomi, Yuko Miura, Hideaki Munemoto, Rice crop field monitoring system with radio controlled helicopter based near infrared cameras through nitrogen content estimation and its distribution monitoring, International Journal of Advanced Research in Artificial Intelligence, 2, 3, 26-37, 2013.

[22] Kohei Arai, Rice crop quality evaluation method through regressive analysis between nitrogen content and near infrared reflectance of rice leaves measured from near field radio controlled helicopter, International Journal of Advanced Research in Artificial Intelligence, 2, 5, 1-6, 2013.

[23] K.Arai, Masanori Sakashita, Osamu Shigetomi, Yuko Miura, Estimation of protein content in rice crop and nitrogen content in rice leaves through regressive analysis with NDVI derived from camera mounted radio-control helicopter, International Journal of Advanced Research in Artificial Intelligence, 3, 3, 7-14, 2014.

[24] Kohei Arai, Masanori Sakashita, Osamu Shigetomi, Yuko Miura, Relation between rice crop quality (protein content) and fertilizer amount as well as rice stump density derived from helicopter data, International Journal of Advanced Research on Artificial Intelligence, 4, 7, 29-34, 2015.

[25] Kohei Arai, Masanori Sakashita, Osamu Shigetomi, Yuko Miura, Estimation of Rice Crop Quality and Harvest Amount from Helicopter Mounted NIR Camera Data and Remote Sensing Satellite Data, International Journal of Advanced Research on Artificial Intelligence, 4, 9,, 2015.

[26] Kohei Arai and Yui Nishimura, Degree of polarization model for leaves and discrimination between pea and rice types leaves for estimation of leaf area index, Abstract of the 50th COSPAR(Committee on Space Research/ICSU) Congress, A3.1-0010-08 ,991, Montreal, Canada, July, 2008 


\section{AUTHORS PROFILE}

Kohei Arai, He received BS, MS and PhD degrees in 1972, 1974 and 1982, respectively. He was with The Institute for Industrial Science and Technology of the University of Tokyo from April 1974 to December 1978 also was with National Space Development Agency of Japan from January, 1979 to March, 1990. During from 1985 to 1987, he was with Canada Centre for Remote Sensing as a Post Doctoral Fellow of National Science and Engineering Research Council of Canada. He moved to Saga University as a
Professor in Department of Information Science on April 1990. He was a councilor for the Aeronautics and Space related to the Technology Committee of the Ministry of Science and Technology during from 1998 to 2000 . He was a councilor of Saga University for 2002 and 2003. He also was an executive councilor for the Remote Sensing Society of Japan for 2003 to 2005 . He is an Adjunct Professor of University of Arizona, USA since 1998. He also is Vice Chairman of the Commission "A" of ICSU/COSPAR since 2008. He wrote 33 books and published 510 journal papers. He is now Editor-in-Chief of IJACSA and IJISA. 